\title{
On the Hodge conjecture for quasi-smooth intersections in toric varieties
}

\section{Ugo Bruzzo ${ }^{1,2,3,4,5} \cdot$ William Montoya ${ }^{6}(\mathbb{0}$}

Accepted: 13 June 2021 / Published online: 6 July 2021

(c) The Author(s) 2021

\begin{abstract}
We establish the Hodge conjecture for some subvarieties of a class of toric varieties. First we study quasi-smooth intersections in a projective simplicial toric variety, which is a suitable notion to generalize smooth complete intersection subvarieties in the toric environment, and in particular quasi-smooth hypersurfaces. We show that under appropriate conditions, the Hodge conjecture holds for a very general quasi-smooth intersection subvariety, generalizing the work on quasi-smooth hypersurfaces of the first author and Grassi in Bruzzo and Grassi (Commun Anal Geom 28: 1773-1786, 2020). We also show that the Hodge Conjecture holds asymptotically for suitable quasi-smooth hypersurface in the Noether-Lefschetz locus, where "asymptotically" means that the degree of the hypersurface is big enough, under the assumption that the ambient variety $\mathbb{P}_{\Sigma}^{2 k+1}$ has Picard group $\mathbb{Z}$. This extends to a class of toric varieties Otwinowska's result in Otwinowska (J Alg Geom 12: 307320, 2003).
\end{abstract}

Communicated by Kostiantyn Iusenko.

William Montoya

montoya@unicamp.br

Ugo Bruzzo

bruzzo@sissa.it

1 SISSA (Scuola Internazionale Superiore di Studi Avanzati), Via Bonomea 265, 34136 Trieste, Italy

2 Departamento de Matemática, Universidad Federal da Paraíba, Campus I, Jardim Universitário, João Pessoa, PB 58051-900, Brazil

3 INFN (Istituto Nazionale di Fisica Nucleare), Sezione di Trieste, Trieste, Italy

4 IGAP (Institute for Geometry and Physics), Trieste, Italy

5 Arnold-Regge Center for Algebra, Geometry and Theoretical Physics, Turin, Italy

6 Instituto de Matemática, Estatística e Computação Científica, Universidade Estadual de Campinas, Rua Sérgio Buarque de Holanda 651, Campinas, SP 13083-859, Brazil 
Keywords Noether-Lefschetz theory $\cdot$ Hodge conjecture $\cdot$ Toric varieties

Mathematics Subject Classification $14 \mathrm{C} 22 \cdot 14 \mathrm{~J} 70 \cdot 14 \mathrm{M} 25$

\section{Introduction}

A projective simplicial toric variety $\mathbb{P}_{\Sigma}^{d}$ satisfies the Hodge Conjecture, i.e., every cohomology class in $H^{p, p}\left(\mathbb{P}_{\Sigma}^{d}, \mathbb{Q}\right)$ is a linear combination of algebraic cycles. On the one hand, by the Lefschetz hyperplane theorem, the Hodge conjecture holds true for every hypersurface and $p<\frac{d-1}{2}$ and by the hard Lefschetz theorem also for $p>\frac{d-1}{2}$. Moreover, by Theorem 1.1 in [3], when $p=\frac{d-1}{2}, d=2 k+1$ and $\mathbb{P}_{\Sigma}^{2 k+1}$ is an Oda variety with an ample class $\beta$ such that $k \beta-\beta_{0}$ is nef, where $\beta_{0}$ is the anticanonical class, the Hodge conjecture with rational coefficients holds for a very general hypersurface in the linear system $|\beta|$.

The notion of Oda varieties was introduced in [2]. Let us recall that the Cox ring of a toric variety $\mathbb{P}_{\Sigma}$ is graded over the class group $\mathrm{Cl}\left(\mathbb{P}_{\Sigma}\right)$, and that one has an injection $\operatorname{Pic}\left(\mathbb{P}_{\Sigma}\right) \rightarrow \operatorname{Cl}\left(\mathbb{P}_{\Sigma}\right)$.

Definition 1.1 Let $\mathbb{P}_{\Sigma}$ be a toric variety with Cox ring $S . \mathbb{P}_{\Sigma}$ is said to be an Oda variety if the multiplication morphism $S^{\alpha_{1}} \otimes S^{\alpha_{2}} \rightarrow S^{\alpha_{1}+\alpha_{2}}$ is surjective whenever the classes $\alpha_{1}$ and $\alpha_{2}$ in $\operatorname{Pic}\left(\mathbb{P}_{\Sigma}\right)$ are ample and nef, respectively.

In [15] Mavlyutov proved a Lefschetz type theorem for quasi-smooth intersection subvarieties, and moreover using the "Cayley trick" he related the cohomology of a quasi-smooth subavariety $X=X_{f_{1}} \cap \cdots \cap X_{f_{s}} \subset \mathbb{P}_{\Sigma}^{d}$ to the cohomology of a quasismooth hypersurface $Y \subset \mathbb{P}_{\Sigma}^{d+s-1}$. This allows us to prove a Noether-Lefschetz type theorem, namely:

Theorem 2.5. Let $\mathbb{P}_{\Sigma}^{d}$ be an Oda projective simplicial toric variety. For a very general quasi-smooth intersection subvariety $X$ cut off by $f_{1}, \ldots f_{s}$ such that $d+s=2(\ell+1)$ and

$$
\sum_{i=1}^{s} \operatorname{deg}\left(f_{i}\right)-\beta_{0}
$$

is nef, one has

$$
H^{\ell+1-s, \ell+1-s}(X, \mathbb{Q})=i^{*}\left(H^{\ell+1-s, \ell+1-s}\left(\mathbb{P}_{\Sigma}^{d}, \mathbb{Q}\right)\right) .
$$

From this one obtains the following result about the Hodge conjecture for quasismooth intersections.

Corollary 2.7. If $\mathbb{P}_{\Sigma}^{d}$ is an Oda projective simplicial toric variety, the Hodge Conjecture holds for a very general quasi-smooth intersection subvariety $X$ cut off by $f_{1}, \ldots f_{s}$ such that $d+s$ is even and $\sum_{i=1}^{s} \operatorname{deg}\left(f_{i}\right)-\beta_{0}$ is nef.

Let $T$ be the open subset of $|\beta|$ corresponding to quasi-smooth hypersurfaces, and let $\mathcal{H}^{2 k}=R^{2 k} \pi_{*} \mathbb{C} \otimes_{\mathbb{C}} \mathcal{O}_{T}$ be the Hodge bundle on $T$; here $\pi: \mathcal{X} \rightarrow T$ is the 
tautological family on $T$, and $d=2 k+1$. We restrict $\mathcal{H}^{2 k}$ to a contractible open subset $U \subset T$. The bundle $\mathcal{H}^{2 k}$ has a Hodge decomposition

$$
\mathcal{H}^{2 k}=\bigoplus_{p+q=2 k} \mathcal{H}^{p, q}
$$

but this is not holomorphic. On the other hand, the bundles that make up the Hodge filtration

$$
F^{p} \mathcal{H}^{2 k}=\bigoplus_{p=0}^{2 k} \mathcal{H}^{2 k-p, p}
$$

are holomorphic; to see this one can use the period map (which in particular we write for $p=k$ )

$$
\mathcal{P}^{k, 2 k}: U \rightarrow \operatorname{Grass}\left(b_{k}, H^{2 k}\left(X_{u_{0}}, \mathbb{C}\right)\right)
$$

where $b_{k}=\operatorname{dim} F^{k} H^{2 k}\left(X_{u_{0}}, \mathbb{C}\right)$ for a fixed point $u_{0} \in U$; this map sends $f \in U$ to the subspace $F^{k} H^{2 k}\left(X_{f}, \mathbb{C}\right) \subset H^{2 k}\left(X_{f}, \mathbb{C}\right)=H^{2 k}\left(X_{u_{0}}, \mathbb{C}\right)$. This map is holomorphic (see [14] and [5, Prop. 3.4]). But, by the very definition of the period map (see also [17], Section 10.2.1 for the smooth case)

$$
F^{k} \mathcal{H}^{2 k} \simeq\left(\mathcal{P}^{k, 2 k}\right)^{*} \mathcal{U}_{k}
$$

where $\mathcal{U}_{k}$ is the tautological bundle on the Grassmannian $\operatorname{Grass}\left(b_{k}, H^{2 k}\left(X_{u_{0}}, \mathbb{C}\right)\right)$, so that the bundles $F^{k} \mathcal{H}^{2 k}$ are indeed holomorphic.

Pushing ahead the ideas developed in [5] and [4], let $\lambda_{f}$ be a nonzero class in the primitive cohomology $H^{k, k}\left(X_{f}, \mathbb{Q}\right) / H^{k, k}\left(\mathbb{P}_{\Sigma}^{2 k+1}, \mathbb{Q}\right)$, and let $U$ be a contractible open subset of $T$ around $f$, so that $\mathcal{H}_{\mid U}^{2 k}$ is constant. Moreover, let $\lambda \in \mathcal{H}^{2 k}(U)$ be the section defined by $\lambda_{f}$ and let $\bar{\lambda}$ be its image in $\left(\mathcal{H}^{2 k} / F^{k} \mathcal{H}^{2 k}\right)(U)$. One has

Proposition 1.2 The local Noether-Lefschetz loci can be defined as

$$
N_{\lambda, U}^{k, \beta}:=\left\{G \in U \mid \bar{\lambda}_{G}=0\right\}
$$

where $\beta=\operatorname{deg}(f)$.

The following result is Theorem 1.2 in [4].

Theorem. Let $\mathbb{P}_{\Sigma}^{2 k+1}$ be an Oda variety with an ample class $\beta$ such that $k \beta-\beta_{0}=n \eta$, where $\beta_{0}$ is the anticanonical class, $\eta$ is a primitive ample class, and $n \in \mathbb{N}$. Let

$$
m_{\beta}=\max \{i \in \mathbb{N} \mid i \eta \leq \beta\} .
$$

For every positive $\epsilon$ there is a positive $\delta$ such that for every $m \geq \max \left(\frac{1}{\delta}, m_{\beta}\right)$ and $\hat{d} \in[1, m \delta]$, and every nontrivial Hodge class $\lambda \in F^{k} \mathcal{H}^{2 k}(U)$ such that 


$$
\operatorname{codim} N_{\lambda, U}^{k, \beta} \leq \hat{d} \frac{m_{\beta}^{k}}{k !}
$$

for every $f \in N_{\lambda, U}^{k, \beta}$, there exists a $k$-dimensional variety $V \subset X_{f}$ with $\operatorname{deg} V \leq(1+\epsilon) \hat{d}$. Here $\operatorname{deg} V$ is taken with respect to the ample divisor $\eta$, i.e.,

$$
\operatorname{deg} V=[V] \cdot \eta^{k} .
$$

Based on this, in this paper we obtain the following result.

Theorem 4.2. Under the same hypotheses of the previous theorem, assume also that $\operatorname{Pic}\left(\mathbb{P}_{\tau}^{2 k+1}\right)=\mathbb{Z}$. Then, if $V \subset X_{f}$ is a nonempty quasi-smooth intersection subvariety of $\mathbb{P}_{\Sigma}^{2 k+1}$ for some $f \in N_{\lambda, U}^{k, \beta}$, there exists $c \in \mathbb{Q}^{*}$ such that $\lambda_{f}=c \lambda_{V}$, where $\lambda_{V}$ is the class of $V$ in $H_{\text {prim }}^{k, k}\left(X_{f}, \mathbb{Q}\right)$.

In other words, $\lambda_{f}$ is algebraic.

In his paper [11] A. Dan proves a form of our Theorem 4.2 for smooth hypersurfaces in odd-dimensional projective spaces $\mathbb{P}^{2 k+1}$ which is not asymptotic. Although our result is more general in two ways, as we consider quasi-smooth intersections in toric varieties with $h^{k, k}=1$ (for instance, weighted or fake projective spaces); however, our result is asymptotic.

In Sect. 3 we give an extension of the notion of Gorenstein ideal to Cox rings; this may have some interest on its own.

\section{Very general quasi-smooth intersections}

Let $f_{1}, \ldots, f_{s}$ be homogeneous polynomials in the Cox ring $S=\mathbb{C}\left[x_{1}, \ldots, x_{n}\right]$ of $\mathbb{P}_{\Sigma}^{d}$. Their zero locus $V\left(f_{1}, \ldots, f_{s}\right)$ defines a closed subvariety $X \subset \mathbb{P}_{\Sigma}^{d}$. Let $U(\Sigma)=\mathbb{A}^{n}-Z(\Sigma)$, where $Z(\Sigma)$ is the irrelevant locus, i.e., $Z(\Sigma)=\operatorname{Spec} B$, where $B$ is the irrelevant ideal.

Definition 2.1 [15] $X$ is a codimension $s$ quasi-smooth intersection if $V\left(f_{1}, \ldots, f_{s}\right) \cap U(\Sigma)$ is either empty or a smooth intersection subvariety of codimension $s$ in $U(\Sigma)$.

This notion generalizes that of smooth complete intersection in a projective space. For $s=1$ it reduces to the notion of quasi-smoooth hypersurface, see Def. 3.1 in [1]. If we regard $\mathbb{P}_{\Sigma}^{d}$ as an orbifold, then an intersection of hypersurfaces $X_{f_{1}} \cap \cdots \cap X_{f_{s}}$ is quasi-smooth when it is a sub-orbifold of $\mathbb{P}_{\Sigma}^{d}$, see Prop 1.3 [15]; heuristically, " $X$ has only singularities coming from the ambient variety."

We also have a Lefschetz type theorem in this context.

Proposition 2.2 ( [15] Proposition 1.4) Let $X \subset \mathbb{P}_{\Sigma}^{d}$ be a closed subset, defined by homogeneous polynomials $f_{1}, \ldots f_{s} \in B$. Then the natural map $i^{*}: H^{i}\left(\mathbb{P}_{\Sigma}^{d}\right) \rightarrow H^{i}(X)$ is an isomorphism for $i<d-s$ and an injection for $i=d-s$. In particular, this is true if the hypersurfaces cut by the polynomials $f_{i}$ are ample. 
Hence if $p \neq \frac{d-s}{2}$ every cohomology class in $H^{p, p}(X)$ is a linear combination of algebraic cycles. So let us see what happens when $p=\frac{d-s}{2}$. The idea is to relate the Hodge structure of a quasi-smooth intersection variety $X^{2}=X_{f_{1}} \cap \cdots \cap X_{f_{s}}$ in $\mathbb{P}_{\Sigma}^{d}$ with the Hodge structure of a quasi-smooth hypersurface $Y$ in a toric variety $\mathbb{P}_{X, \Sigma}^{d+s-1}$ whose fan depends on $X$ and $\Sigma$.

Proposition 2.3 Let $X=X_{f_{1}} \cap \cdots \cap X_{f_{s}}$ be quasi-smooth intersection subvariety in $\mathbb{P}_{\Sigma}^{d}$ cut off by homogeneous polynomials $f_{1} \ldots f_{s}$. There exists a projective simplicial toric variety $\mathbb{P}_{X, \Sigma}^{d+s-1}$ and a quasi-smooth hypersurface $Y \subset \mathbb{P}_{X, \Sigma}^{d+s-1}$ such that for $p \neq \frac{d+s-1}{2}, \frac{d+s-3}{2}$

$$
H_{\text {prim }}^{p-1, d+s-1-p}(Y) \simeq H_{\text {prim }}^{p-s, d-p}(X) .
$$

Proof One constructs $\mathbb{P}_{X, \Sigma}^{d+s-1}$ via the so-called "Cayley trick". Let $L_{1}, \ldots, L_{s}$ be the line bundles associated to the quasi-smooth hypersurfaces $X_{1}, \ldots X_{s}$, and so let $\mathbb{P}(E)$ be the projective bundle of $E=L_{1} \oplus \cdots \oplus L_{s}$. It turns out that $\mathbb{P}(E)$ is a $d+s-1$ dimensional projective simplicial toric variety whose Cox ring is

$$
\mathbb{C}\left[x_{1}, \ldots, x_{n}, y_{1}, \ldots y_{s}\right]
$$

where $S=\mathbb{C}\left[x_{1}, \ldots, x_{n}\right]$ is the Cox ring of $\mathbb{P}_{\Sigma}^{d}$. The hypersurface $Y$ is cut off by the polynomial $F=y_{1} f_{1}+\cdots+y_{s} f_{s}$ and is quasi-smooth by Lemma 2.2 in [15]. Moreover, combining Theorem 10.13 in [1] and Theorem 3.6 in [15], we have that

$$
H_{\text {prim }}^{p-1, d+s-1-p}(Y) \simeq R(F)_{(d+s-p) \beta-\beta_{0}} \simeq H_{\text {prim }}^{p-s, d-p}(X)
$$

for $p \neq \frac{d+s-1}{2}, \frac{d+s-3}{2}$ as desired.

Here $R(F)$ is the Jacobian ring of $Y$, i.e., the quotient of the Cox ring

$$
R(F)=\mathbb{C}\left[x_{1}, \ldots, x_{n}, y_{1}, \ldots y_{s}\right] / J(F),
$$

where $J(F)$ is the ideal generated by the derivatives of $F$, see [1].

Remark 2.4 With the same notation of Proposition 2.3, note that we have a well defined map

$$
\begin{aligned}
\phi:\left|\beta_{1}\right| \times \cdots \times\left|\beta_{s}\right| & \rightarrow|\beta| \\
\left(f_{1}, \ldots, f_{s}\right) & \mapsto f_{1} y_{1}+\cdots+f_{s} y_{s} .
\end{aligned}
$$

Moreover, by the Noether-Lefschetz theorem $N L_{\beta}$ is a countable union of closed sets $\bigcup_{i} C_{i}$ and hence $\bigcup \phi^{-1}\left(C_{i}\right)$ is too.

We have a Noether-Lefschetz type theorem, namely, 
Theorem 2.5 Let $\mathbb{P}_{\Sigma}^{d}$ be an Oda projective simplicial toric variety. Then for a very general quasi-smooth intersection subvariety $X$ cut off by $f_{1}, \ldots f_{s}$ such that $d+s=2(l+1)$ and $\sum_{i=1}^{s} \operatorname{deg}\left(f_{i}\right)-\beta_{0}$ is nef, one has that

$$
H^{l+1-s, l+1-s}(X, \mathbb{Q})=i^{*}\left(H^{l+1-s, l+1-s}\left(\mathbb{P}_{\Sigma}^{d}, \mathbb{Q}\right)\right)
$$

So we get a natural generalization of the Noether-Lefschetz loci.

Definition 2.6 The Noether-Lefschetz locus $N L_{\beta_{1}, \ldots, \beta_{s}}$ of quasi-smooth intersection varieties is the locus of $s$-tuples $\left(f_{1}, \ldots, f_{s}\right)$ such that $X=X_{f_{1}} \cap \ldots X_{f_{s}}$ is quasi-smooth intersection with $f_{i} \in\left|\beta_{i}\right|$ and $H^{l+1-s, l+1-s}(X, \mathbb{Q}) \neq i^{*}\left(H^{l+1-s, l+1-s}\left(\mathbb{P}_{\Sigma}^{d}, \mathbb{Q}\right)\right)$.

Now we consider the Hodge conjecture for very general quasi-smooth intersection subvarieties in $\mathbb{P}_{\Sigma}^{d}$.

Corollary 2.7 If $\mathbb{P}_{\Sigma}^{d}$ is a Oda projective simplicial toric variety, the Hodge Conjecture holds for a very general quasi-smooth intersection subvariety $X$ cut off by $f_{1}, \ldots f_{s}$ such that $d+s=2(l+1)$ and $\sum_{i=1}^{s} \operatorname{deg}\left(f_{i}\right)-\beta_{0}$ is nef.

Proof First note that by Thereom 4.1 in [12] the projective simplicial toric variety $\mathbb{P}_{X, \Sigma}^{2 l+1}$ is Oda and since $X$ is very general the quasi-smooth hypersurface $Y$ is very general as well. So applying the Noether-Lefschetz theorem one has that $h_{\text {prim }}^{l, l}(Y)=0=h_{\text {prim }}^{l+1-s, l+1-s}(X)$ or equivalently every $(l+1-s,+1-s)$ cohomology class is a linear combination of algebraic cycles.

\section{Cox-Gorenstein ideals}

We shall need a partial generalization of Macaulay's theorem (see e.g. Thm. 6.19 in [18] for the classical theorem). This generalization is basically contained in the work of Cox and Cattani-Cox-Dickenstein [7, 9].

Let $S$ be the Cox ring of a complete simplicial toric variety $\mathbb{P}_{\Sigma}$. This is graded over the effective classes in the class group $\mathrm{Cl}\left(\mathbb{P}_{\Sigma}\right)$ and [8]

$$
S^{\alpha} \simeq H^{0}\left(\mathbb{P}_{\Sigma}, \mathcal{O}_{\mathbb{P}_{\Sigma}}(\alpha)\right)
$$

As $\mathcal{O}_{\mathbb{P}_{\Sigma}}(\alpha)$ is coherent and $\mathbb{P}_{\Sigma}$ is complete, each $S^{\alpha}$ is finite-dimensional over $\mathbb{C}$; in particular, $S^{0} \simeq \mathbb{C}$.

Lemma 3.1 For every effective $N \in \mathrm{Cl}\left(\mathbb{P}_{\Sigma}\right)$, the set of classes $\alpha \in \mathrm{Cl}\left(\mathbb{P}_{\Sigma}\right)$ such that $N-\alpha$ is effective is finite.

Proof Since the torsion submodule of $\mathrm{Cl}\left(\mathbb{P}_{\Sigma}\right)$ is finite, we may assume that $\mathrm{Cl}\left(\mathbb{P}_{\Sigma}\right)$ is free. Then the exact sequence 


$$
0 \rightarrow M \rightarrow \operatorname{Div}_{\mathbb{T}}\left(\mathbb{P}_{\Sigma}\right) \rightarrow \mathrm{Cl}\left(\mathbb{P}_{\Sigma}\right) \rightarrow 0
$$

splits, and we may identify $\mathrm{Cl}\left(\mathbb{P}_{\Sigma}\right)$ with a free subgroup of $\operatorname{Div}_{\mathbb{T}}\left(\mathbb{P}_{\Sigma}\right)$, generated by a subset $\left\{D_{1}, \ldots, D_{r}\right\}$ of $\mathbb{T}$-invariant divisors. A class in $\mathrm{Cl}\left(\mathbb{P}_{\Sigma}\right)$ is effective if and only its coefficients on this basis are nonnegative, whence the claim follows.

We shall give a definition of Cox-Gorenstein ideal of the Cox rings which generalizes to toric varieties the definition given by Otwinowska in [16] for projective spaces. Let $B \subset S$ be the irrelevant ideal, and for a graded ideal $I \subset B$, denote by $V_{\mathbb{T}}(I)$ the corresponding closed subscheme of $\mathbb{P}_{\Sigma}$.

Definition 3.2 A graded ideal I of $S$ contained in B is said to be a Cox-Gorentstein ideal of socle degree $N \in \mathrm{Cl}\left(\mathbb{P}_{\Sigma}\right)$ if

1. there exists a $\mathbb{C}$-linear form $\Lambda \in\left(S^{N}\right)^{\vee}$ such that for all $\alpha \in \operatorname{Cl}\left(\mathbb{P}_{\Sigma}\right)$

$$
I^{\alpha}=\left\{f \in S^{\alpha} \mid \Lambda(f g)=0 \text { for all } g \in S^{N-\alpha}\right\}
$$

2. $V_{\mathbb{T}}(I)=\emptyset$.

Remark 3.3 Cox-Gorenstein ideals need not be Artinian. Property 2 in this definition replaces that condition.

Proposition 3.4 Let $R=S / I$. If I is Cox-Gorenstein then

1. $\operatorname{dim}_{\mathbb{C}} R^{N}=1$;

2. the natural bilinear morphism

$$
R^{\alpha} \times R^{N-\alpha} \rightarrow R^{N} \simeq \mathbb{C}
$$

is nondegenerate whenever $\alpha$ and $N-\alpha$ are effective.

\section{Proof}

1. From eq. (2) we see that the sequence

$$
0 \rightarrow I^{N} \rightarrow S^{N} \stackrel{\Lambda}{\rightarrow} \mathbb{C} \rightarrow 0
$$

is exact.

2. Define $\Phi: R^{\alpha} \times R^{N-\alpha} \rightarrow \mathbb{C}$ as $\Phi(x, y)=\Lambda(\bar{x} \bar{y})$, where $\bar{x}, \bar{y}$ are pre-images of $x$, $y$ in $S$. One easily checks that this is well defined and that via the isomorphism $R^{N} \simeq k$ it coincides with the pairing (3). Now if $x \in R^{\alpha}$ and $\Phi(x, y)=0$ for all $y \in R^{N-\alpha}$ then $\Lambda(\bar{x} \bar{y})=0$ for all $\bar{y} \in S^{N-\alpha}$ so that $\bar{x} \in I^{\alpha}$, i.e., $x=0$. 
Let $f_{0}, \ldots, f_{d}$ be homogeneous polynomials, $f_{i} \in S^{\alpha_{i}}$, where $d=\operatorname{dim} \mathbb{P}_{\Sigma}$ and each $\alpha_{i}$ is ample, and let $N=\sum_{i} \alpha_{i}-\beta_{0}$, where $\beta_{0}$ is the anticanonical class of $\mathbb{P}_{\Sigma}$. Assume that the $f_{i}$ have no common zeroes in $\mathbb{P}_{\Sigma}$, i.e., $V_{\mathbb{T}}(I)=\emptyset$ if $I=\left(f_{0}, \ldots, f_{d}\right)$.

In $[1,7,9]$ it is shown that for each $G \in S^{N}$ one can define a meromorphic $d$-form $\xi_{G}$ on $\mathbb{P}_{\Sigma}$ by letting

$$
\xi_{G}=\frac{G \Omega}{f_{0} \cdots f_{d}}
$$

where $\Omega$ is a Euler form on $\mathbb{P}_{\Sigma}$. The form $\xi_{G}$ determines a class in $H^{d}\left(\mathbb{P}_{\Sigma}, \omega\right)$, where $\omega$ is the canonical sheaf of $\mathbb{P}_{\Sigma}$ (the sheaf of Zariski $d$-forms on $\mathbb{P}_{\Sigma}$ ), and in turn the trace morphism $\operatorname{Tr}_{\mathbb{P}_{\Sigma}}: H^{d}\left(\mathbb{P}_{\Sigma}, \omega\right) \rightarrow \mathbb{C}$ associates a complex number to $G$, so we can define $\Lambda \in\left(S^{N}\right)^{\vee}$ as

$$
\Lambda(G)=\operatorname{Tr}_{\mathbb{P}_{\Sigma}}\left(\left[\xi_{G}\right]\right) \in \mathbb{C} .
$$

Finally, we can prove a toric version of Macaulay's theorem.

Theorem 3.5 The linear map defined in Eq. (4) satisfies the condition in Definition 3.2. Therefore, the ideal $I=\left(f_{0}, \ldots, f_{d}\right)$ is a Cox-Gorenstein ideal of socle degree $N$.

Proof By Theorem 6 in [7] the map $\Lambda$ establishes an isomorphism $R^{N} \simeq \mathbb{C}$. Hence, if $f \in S^{\alpha}$ is such that $\Lambda(f g)=0$ for all $g \in S^{N-\alpha}$, then $f g \in I^{N}$, which implies $f \in I^{\alpha}$. On the other hand, it is clear that $\Lambda(f g)=0$ if $f \in I^{\alpha}$ and $g \in S^{N-\alpha}$.

Another example is given in terms of toric Jacobian ideals. For every ray $\rho \in \Sigma(1)$ we shall denote by $v_{\rho}$ its rational generator, and by $x_{\rho}$ the corresponding variable in the Cox ring. Recall that $d$ is the dimension of the toric variety $\mathbb{P}_{\Sigma}$, while we denote by $r=\# \Sigma(1)$ the number of rays. Given $f \in S^{\beta}$ one defines its toric Jacobian ideal as

$$
J_{0}(f)=\left(x_{\rho_{1}} \frac{\partial f}{\partial x_{\rho_{1}}}, \ldots, x_{\rho_{r}} \frac{\partial f}{\partial x_{\rho_{r}}}\right) .
$$

We recall from [1] the definition of nondegenerate hypersurface and some properties (Def. 4.13 and Prop. 4.15).

Definition 3.6 Let $f \in S(\Sigma)^{\beta}$, with $\beta$ an ample Cartier class. The associated hypersurface $X_{f}$ is nondegenerate if for all $\sigma \in \Sigma$ the affine hypersurface $X_{f} \cap O(\sigma)$ is a smooth codimension one subvariety of the orbit $O(\sigma)$ of the action of the torus $\mathbb{T}^{d}$.

\section{Proposition 3.7}

1. Every nondegenerate hypersurface is quasi-smooth.

2. If $f$ is generic then $X_{f}$ is nondegenerate.

The following is part of Prop. 5.3 in [9], with some changes in the terminology. 
Proposition 3.8 Let $f \in S(\Sigma)^{\beta}$, and let $\left\{\rho_{1}, \ldots, \rho_{d}\right\} \subset \Sigma(1)$ be such that $v_{\rho_{1}}, \ldots, v_{\rho_{d}}$ are linearly independent.

1. The toric Jacobian ideal of f coincides with the ideal

$$
\left(f, x_{\rho_{1}} \frac{\partial f}{\partial x_{\rho_{1}}}, \ldots, x_{\rho_{d}} \frac{\partial f}{\partial x_{\rho_{d}}}\right) .
$$

2. The following conditions are equivalent:

(a) $f$ is nondegenerate;

(b) the polynomials $x_{\rho_{i}} \frac{\partial f}{\partial x_{\rho_{i}}}, i=1, \ldots, r$, do not vanish simultaneously on $X_{f}$;

(c) the polynomials $f$ and $x_{\rho_{i}} \frac{\partial f}{\partial x_{\rho_{i}}}, i=1, \ldots, d$, do not vanish simultaneously on $X_{f}$.

3. If moreover $\beta$ is ample and $f$ is nondegenerate, then $J_{0}(f)$ is a Cox-Gorenstein ideal of socle degree $N=(d+1) \beta-\beta_{0}$, where $\beta_{0}$ is the anticanonical class of $\mathbb{P}_{\Sigma}^{d}$.

\section{Asymptotic Hodge conjecture}

In this section we prove Theorem 4.2. Let us recall part of the notation and assumptions of [4]. Let $\mathbb{P}_{\Sigma}^{2 k+1}$ be an Oda variety with an ample Cartier class $\beta$ such that $k \beta-\beta_{0}=n \eta$, where $\beta_{0}$ is the anticanonical class, $\eta$ is a primitive ample class and $n \in \mathbb{N}$.

We need to define a pre-order in the group

$$
N^{1}\left(\mathbb{P}_{\Sigma}^{2 k+1}\right)=\operatorname{Pic}\left(\mathbb{P}_{\Sigma}^{2 k+1}\right) \otimes \mathbb{Q} / \text { numerical equivalence, }
$$

by letting $\alpha<\alpha^{\prime}$ if $\alpha^{\prime}-\alpha$ is an effective class.

Let $X_{f} \in|\beta|$ be a quasi-smooth hypersurface in the Noether-Lefschetz locus associated to a nontrival Hodge class $\lambda \in F^{k} \mathcal{H}^{2 k}(U)$. Again, its degree is computed by intersecting with the ample class $\eta$, i.e., $\operatorname{deg} X_{f}=\left[X_{f}\right] \cdot \eta$. Let $r$ be number of rays of $\Sigma$, so that $r \geq 2(k+1)$. Assuming that $n$ is big enough, it follows from Proposition 4.7 or Theorem 6.1 in [4] that there exists a $k$-dimensional subvariety $V$ of $X_{f}$ satisfying the following conditions:

- $\operatorname{deg} V \leq 2 \delta m_{\beta}$ with $0<\delta<\frac{1}{4(r-(k+1))}$ (the number $m_{\beta}$ was defined in Eq. (1));

- the graded ideals $I_{V}$ and

$$
E=\left\{g \in S^{\bullet} \mid \sum_{i=1}^{b} \lambda_{i} \int_{\mathrm{Tub}_{i}} \frac{g h \Omega_{0}}{f^{k+1}}=0 \text { for all } h \in S^{N-\bullet}\right\},
$$


coincide in degree less than or equal to $\left(m_{\beta}-2-(r-j) \operatorname{deg} V\right) \eta$ for some $j$, with $0<j<r$. Here $\operatorname{Tub}(-)$ is the adjoint of the residue map, and $N=(k+1) \beta-\beta_{0}$ is the socle degre of the Cox-Gorenstein ideal E, while

$$
\lambda_{f}=\left(\sum_{i=1}^{b} \lambda_{i} \gamma_{i}\right)^{p d}
$$

is the Poincaré dual of some rational combination of the homology cycles $\gamma_{i}$ generating $H_{2 k}\left(X_{f}, \mathbb{Q}\right)$. Moreover, via the isomorphism $T_{f} U \simeq S^{\beta}$, the degree $\beta$ summand $E^{\beta}$ of $E$ is identified with the tangent space $T_{f} N_{\lambda, U}^{k, \beta}$ to the Noether-Lefschetz locus, so that $E^{\beta}$ contains the degree $\beta$ part $J(f)^{\beta}$ the Jacobian ideal of $f$.

Lemma 4.1 The toric Jacobian ideal $J_{0}(f)$ is contained in E.

Proof $J_{0}(f) \subset J(f)$, so that $J_{0}(f)^{\beta} \subset J(f)^{\beta} \subset E^{\beta}$, and since $J_{0}(f)$ is generated in degree $\beta$, one has $J_{0}(f) \subset E$.

We denote by $\lambda_{V}$ the class of $V$ in $H_{\text {prim }}^{k, k}\left(X_{f}, \mathbb{Q}\right)$. In the following theorem we assume that $\operatorname{Pic}\left(\mathbb{P}_{\Sigma}^{2 k+1}\right)=1$, i.e., that $\mathbb{P}_{\Sigma}^{2 k+1}$ is a (possibly fake) weighted projective space $[6,13]$ (cf. [10] Exer. 5.1.13). This implies that $h^{p, p}\left(\mathbb{P}_{\Sigma}^{2 k+1}\right)=1$ for all $p$.

Theorem 4.2 If $V$ is a quasi-smooth intersection subvariety, there exists $c \in \mathbb{Q}^{*}$ such that $\lambda_{f}=c \lambda_{V}$.

Proof We divide the proof in three steps.

Step I: $\lambda_{V} \neq 0$. For clarity, for every cohomology class of a subvariety we denote in the cohomology of which ambient variety we consider it (so we write $[V]_{X_{f}}$ and $[V]_{\mathbb{P}_{\Sigma}^{2 k+1}}$. Since $V \subset X_{f}$ is a regular embedding we have

$$
\begin{aligned}
{[V]_{X_{f}}^{2} } & =\int_{V} c_{k}\left(N_{V / X_{f}}\right)=\int_{V}\left[c\left(N_{V / \mathbb{P}_{\Sigma}^{2 k+1}}\right) / c\left(N_{X_{f} / \mathbb{P}_{\Sigma}^{2 k+1} \mid V}\right)\right]_{k} \\
{[8 p t] } & =\int_{\mathbb{P}_{\Sigma}^{2 k+1}}[V]_{\mathbb{P}_{\Sigma}^{2 k+1}} \cup \Xi_{k}
\end{aligned}
$$

where $\Xi_{k}$ is the component in $H^{k, k}\left(\mathbb{P}_{\Sigma}^{2 k+1}\right)$ of

$$
\Xi=\frac{\prod_{i}\left(1+A_{i}\right)}{1+\left[X_{f}\right]_{\mathbb{P}_{\Sigma}^{2 k+1}}} ;
$$

here $A_{1}, \ldots, A_{k+1}$ are the classes in $\mathrm{Cl}\left(\mathbb{P}_{\Sigma}^{2 k+1}\right)$ of the hypersurfaces that cut the quasismooth intersection subvariety $V$. The claim is proved by contradiction: if $[V]_{X_{f}}$ is the restriction of a class in $H^{k, k}\left(\mathbb{P}_{\Sigma}^{2 k+1}\right)$, i.e.,

$$
[V]_{X_{f}}=b \eta_{\mid X_{f}}^{k}
$$

for some $b$, then comparing this with (6) we obtain 


$$
\operatorname{deg} V=m_{k} \operatorname{deg} X_{f},
$$

where $m_{k}$ is defined by $\Xi_{k}=m_{k} \eta^{k}$. But (7) cannot be true when $\operatorname{deg} X_{f}$ is big enough.

Step II. Let $E_{\text {alg }}$ and $E$ be the Cox-Gorenstein ideals associated to $\lambda_{V}$ and $\lambda_{f}$, respectively, as in Eq. (5). To prove the theorem it is enough to show that $E=E_{\text {alg }}$. Note that $I_{V}+J_{0}(f)$ is contained in $E$ and $E_{\text {alg. }}$. Moreover, since $V \subset X_{f}$, and $f$ is quasi-smooth, there exist $K_{1}, \ldots K_{k+1} \in B$ such that $f=A_{1} K_{1}+\ldots A_{k+1} K_{k+1}$ and $\left(A_{1}, \ldots, A_{k+1}, K_{1}, \ldots K_{k+1}\right)$ is a Cox-Gorenstein ideal with socle degree $N$; this will follow from the next step, which concludes the proof.

Step III. It is enough to show that every Cox-Gorenstein ideal $\mathcal{I}$ of socle degree $N$ containing $I_{V}+J_{0}(f)$ also contains $\left(A_{1}, \ldots, A_{k+1}, K_{1}, \ldots K_{k+1}\right)$. By assumption

$$
\left(A_{j}, j \in\{1, \ldots, k+1\}, \sum_{j=1}^{k+1} x_{i} \frac{\partial A_{j}}{\partial x_{i}} K_{j}, i \in 1, \ldots, r\right) \subset \mathcal{I} .
$$

Let us see that $K_{j} \in \mathcal{I}$ for every $j \in\{1, \ldots, k+1\}$. Let $M \in \operatorname{Mat}(r \times(k+1))$ be the matrix $\left[x_{i} \frac{\partial A_{j}}{\partial x_{i}}\right]$ and $K$ the column $\left(K_{j}\right)_{j \in\{1, \ldots, k+1\}}$. Let $I \subset\{1, \ldots r\}$ with cardinality $k+1$ and let $M_{I}$ be the matrix obtained extracting the $i \in I$-arrows of $M$. We have that $\sum_{j=1}^{k+1} x_{i} \frac{\partial A_{j}}{\partial x_{i}} K_{j}=(M K)_{i}=\left(M_{I} K\right)_{i}$; multiplying by the adjoint of $M_{I}$ we get that $\operatorname{det}\left(M_{I}\right) K_{j} \in \mathcal{I}$ for all $j \in\{1, \ldots k+1\}$. On one hand the ideal $\left(\mathcal{I}: K_{j}\right)$ contains the ideal

$$
\mathcal{J}=I_{V}+\left\langle\operatorname{det} M_{I} \mid I \subset\{1, \ldots, r\}, \# I=k+1\right\rangle .
$$

Since $V$ is a smooth complete intersection subvariety, it follows that $\mathcal{J}$ is base point free, and therefore it contains a complete intersection Cox-Gorenstein ideal $\mathcal{J}$ by the toric Macaulay theorem, Theorem 3.5. Since $\mathcal{J}$ is generated in degree less than or equal to $(\operatorname{deg} V) \eta$, we can take $\mathcal{J}$ with the same property. It follows that

$$
\operatorname{soc}(\mathcal{J}) \leq 2(k+1)(\operatorname{deg} V) \eta-\beta_{0} \leq 2 r m_{\beta} \delta \eta-\beta_{0} .
$$

On the other hand if $K_{j} \notin \mathcal{I}$ then $\left(\mathcal{I}: K_{j}\right.$ ) contains a Cox-Gorenstein ideal with socle degree

$$
N-\operatorname{deg} K_{j} \geq N-\beta=k \beta-\beta_{0}
$$

then comparing the above two inequalities and keeping in mind that $r \geq 2(k+1)$, we get

$$
\delta \geq \frac{1}{2 r} \geq \frac{1}{4(r-(k+1))}
$$

which is absurd.

On behalf of all authors, the corresponding author states that there is no conflict of interest. 
Acknowledgements We thank Paolo Aluffi for useful discussions, and Antonella Grassi for developing with the first author the foundations on which this work is based. We are very thankful to the referee for her/his very careful reading, and the many suggestions and remarks which allowed us to greatly improve the presentation of this paper. The first author's research is partly supported by PRIN "Geometry of algebraic varieties" and GNSAGA-INdAM. The second author acknowledges support from FAPESP postdoctoral Grant No. 2019/23499-7.

Funding Open access funding provided by Scuola Internazionale Superiore di Studi Avanzati - SISSA within the CRUI-CARE Agreement.

Open Access This article is licensed under a Creative Commons Attribution 4.0 International License, which permits use, sharing, adaptation, distribution and reproduction in any medium or format, as long as you give appropriate credit to the original author(s) and the source, provide a link to the Creative Commons licence, and indicate if changes were made. The images or other third party material in this article are included in the article's Creative Commons licence, unless indicated otherwise in a credit line to the material. If material is not included in the article's Creative Commons licence and your intended use is not permitted by statutory regulation or exceeds the permitted use, you will need to obtain permission directly from the copyright holder. To view a copy of this licence, visit http://creativecommons.org/licen ses/by/4.0/.

\section{References}

1. Batyrev, D.A., Cox, V.V.: On the Hodge structure of projective hypersurfaces in toric varieties. Duke Math. J. 75, 293-338 (1994)

2. Bruzzo, U., Grassi, A.: The Noether-Lefschetz locus of surfaces in toric threefolds, Commun. Contemp. Math. No. 5 (2017) 1750070 (20 pages)

3. Bruzzo, U., Grassi, A.: On the Hodge conjecture for hypersurfaces in toric varieties. Commun. Anal. Geom. 28, 1773-1786 (2020)

4. Bruzzo, U., Montoya, W.D.: An asymptotic description of the Noether-Lefschetz components in toric varieties, arXiv:1905.01570 [math.AG]

5. Bruzzo, U., Montoya, W.D.: Codimension bounds for the Noether-Lefschetz components for toric varieties, arXiv:2001.01960 [math.AG]. To appear in Eur. J. Math

6. Buczyńska, W.: Fake weighted projective spaces, English translation of Toryczne przestrzenie rzutowe, Magister thesis, (2002). arXiv:0805.1211 [math.AG]

7. Cattani, E., Cox, D., Dickenstein, A.: Residues in toric varieties. Compos. Math. 108, 35-76 (1997)

8. Cox, D.A.: The homogeneous coordinate ring of a toric variety 4, 17-50 (1995)

9. Cox, D.A.: Toric residues. Ark. Mat. 75, 73-96 (1996)

10. Cox, D., Little, J., Schenck, H.: Toric varieties, Graduate Studies in Mathematics, 124. American Mathematical Society, Providence, RI, (2011). xxiv+841 pp

11. Dan, A.: Noether-Lefschetz locus and a special case of the variational Hodge conjecture: using elementary techniques, In: "Analytic and algebraic geometry," pp. 107-115. New Delhi, Hindustan Book Agency (2017)

12. Ikeda, A.: Subvarieties of generic hypersurfaces in a non-singular projective toric variety. Math. Z. 263, 923-937 (2009)

13. Kawasaki, T.: Cohomology of twisted projective spaces and lens complexes. Math. Ann. 206, 243$248(1973)$

14. Liu, K.F., Zhuang, X.B.: Deformations of complex orbifolds and the period maps. Sci. China Math. 63, 83-100 (2020)

15. Mavlyutov, A.: Cohomology of Complete Intersections In Toric Varieties. Pacific J. Math. 191, 133-144 (1999)

16. Otwinowska, A.: Composantes de petite codimension du lieu de Noether-Lefschetz: un argument asymptotique en faveur de la conjecture de Hodge. J. Alg. Geom. 12, 307-320 (2003)

17. Voisin, C.: Hodge Theory and Complex Algebraic Geometry I. Cambridge University Press, Cambridge (2002) 
18. Voisin, C.: Hodge Theory and Complex Algebraic Geometry II. Cambridge University Press, Cambridge, UK (2003)

Publisher's Note Springer Nature remains neutral with regard to jurisdictional claims in published maps and institutional affiliations. 